REPLY

\title{
Further approaches to reduce the cost of renal replacement therapy
}

\author{
Raymond Vanholder, Lieven Annemans, Edwina Brown, Ron Gansevoort, \\ Judith J. Gout-Zwart, Norbert Lameire, Rachael L. Morton, Rainer Oberbauer, \\ Maarten J. Postma, Marcello Tonelli, Wim Van Biesen and Carmine Zoccali \\ on behalf of the European Kidney Health Alliance
}

We read with interest the comment by M. E. Elrggal and R. Zyada (Gradual initiation of dialysis as a means to reduce cost while providing quality health care. Nat. Rev. Nephrol. http://dx.doi.org/10.1038/ nrneph.2017.135; 2017) $)^{1}$ regarding our article discussing how to combat costs of kidney disease care (Vanholder, R. et al. Reducing the costs of chronic kidney disease while delivering quality health care: a call to action. Nat. Rev. Nephrol. 13, 393-409; 2017) ${ }^{2}$.

In their correspondence, the authors add to our proposed approaches to reduce the costs of renal replacement therapy, by suggesting the use of incremental haemodialysis (that is, starting patients on dialysis with one or two sessions per week instead of the habitual three) as another means to reduce financial stress on health-care systems. On the basis of mathematics alone, this approach would undeniably reduce costs. Nevertheless we think this concept should be regarded with care, as stated in a 2014 comment by members of our group ${ }^{3}$ on a position statement propagating this approach ${ }^{4}$.

In looking at the conditions that are required to consider someone for incremental dialysis ${ }^{3,4}$, one might realize that many patients initiating dialysis do not match these criteria, especially if the 2011 recommendations to start dialysis only in symptomatic patients are taken into account ${ }^{5}$. Most of these restrictions indeed refer to patients with symptoms or disorders that lead to conditions necessitating dialysis initiation, such as fluid overload, heart failure, hyperkalaemia, hyperphosphataemia and malnutrition. Hence, each time dialysis is needed to combat fluid overload (and by extension any other symptom or disturbance that would necessitate start of dialysis), restricting the number of sessions might increase the risk of complications due to the irregularity of the treatment scheme (for instance, by having to remove too much plasma water by ultrafiltration or by enhancing the so-called 'saw-tooth pattern' of metabolic markers). On the other hand, if a patient has no symptoms, no intrinsic need to start dialysis exists, not even at one dialysis session per week.

Elrggal and Zyada attribute the high mortality associated with early dialysis to dialysis intensity, but no hard data exist to support this thesis, and we are not aware of any controlled studies that favour incremental dialysis. Of note, the long weekend interval is a notorious cause of dialysis mortality ${ }^{6}$, which is difficult to reconcile with a philosophy propagating even longer intervals, and to our knowledge the only conclusive controlled study on dialysis timeframes showed a survival advantage of increasing, not decreasing, frequency ${ }^{7}$.

Hence, incremental dialysis might be an option for starting haemodialysis, but it should in our opinion be applied in carefully selected patients. In addition, as this option cannot be used in all patients, can only be used for the first months of dialysis, after which residual kidney function declines, and it is very likely that more structural interventions will be needed ${ }^{2}$, one wonders whether societal financial relief will be substantial. How and when to increase the frequency of dialysis sessions in these incremental schemes also remains unclear. We agree that incremental dialysis might be a useful option, especially in low and middle income countries like Egypt. However, we remain convinced that in those countries as well as elsewhere, the options of kidney transplantation, peritoneal dialysis and kidney disease prevention are underexploited, and that it is the responsibility of the nephrological communities around the world to propagate those solutions, which very likely will have more impact on health economics than will incremental dialysis.
Raymond Vanholder, Norbert Lameire and Wim Van Biesen are at Nephrology Section, Department of Internal Medicine, Ghent University Hospital OK12, De Pintelaan 185, B9000, Ghent, Belgium.

Lieven Annemans is at Ghent University, Faculty of Medicine, Department of Public Health, De Pintelaan 185, B9000, Ghent, Belgium.

Edwina Brown is at Imperial College Renal and Transplant Centre, Hammersmith Hospital, Du Cane Road, London, W12 OHS, UK.

Ron Gansevoort is at Department of Nephrology. University Medical Center Groningen, University Hospital Groningen, Hanzeplein, 1, 9713 GZ Groningen, Netherlands.

Judith J. Gout-Zwart and Maarten J. Postma are at Unit of Pharmacotherapy, Epidemiology \& Economics (PTE2), Department of Pharmacy, University of Groningen, Antonius Deusinglaan 1, 9713 AV Groningen, Netherlands.

Rachael L. Morton is at NHMRC Clinical Trials Centre, Sydney Medical School, University of Sydney, 92-94 Parramatta Road, Camperdown, NSW 2050, Australia.

Rainer Oberbauer is at Department of Nephrology $6 \mathrm{~J}$, Internal Medicine III, Währinger Gürtel 18-20, 1090 Vienna, Austria.

Maarten J. Postma is at Department of Epidemiology, University Medical Center Groningen (UMCG), University of Groningen, Hanzeplein 1. 9713 GZ Groningen, Netherlands.

Maarten J. Postma is at Insitute for Science in Healthy Aging \& Healthcare (SHARE), University Medical Center Groningen (UMCG), University of Groningen, Hanzeplein 1, 9713 GZ Groningen, Netherlands.

Marcello Tonelli is at University of Calgary, 7th Floor, TRW Building, 3280 Hospital Drive NW, Calgary, Alberta T2N 4Z6, Canada.

Carmine Zoccali is at CNR-IFC, Clinical Epidemiology and Pathophysiology of Hypertension and Renal Diseases Unit, Ospedali Riuniti, 89124 Reggio Calabria, Italy.

Correspondence to R.V. raymond.vanholder@ugent.be doi:10.1038/nrneph.2017.136 Published online 25 Sep 2017

. Elrggal, M. E. \& Zyada, R. Gradual initiation of dialysis as a means to reduce cost while providing quality health care. Nat. Rev. Nephrol. http://dx.doi.org/ 10.1038/nrneph.2017.135 (2017).

2. Vanholder, R. et al. Reducing the costs of chronic kidney disease while delivering quality health care: call to action. Nat. Rev. Nephrol. 13, 393-409 (2017). 3. Vanholder, R., Van Biesen, W. \& Lameire, N. Is starting hemodialysis on a twice-weekly regimen a valid option? Am. J. Kidney Dis. 64, 165-167 (2014).

4. Kalantar-Zadeh, K. et al. Twice-weekly and incremental hemodialysis treatment for initiation of kidney replacement therapy. Am. J. Kidney Dis. 64, 181-186 (2014).

5. Tattersall, J. et al. When to start dialysis: updated guidance following publication of the Initiating Dialysis Early and Late (IDEAL) study. Nephrol. Dial. Transplant. 26, 2082-2086 (2011).

6. Bleyer, A. J. et al. Characteristics of sudden death in hemodialysis patients. Kidney Int. 69, 2268-2273 (2006).

7. Chertow, G. M. et al. In-center hemodialysis six times per week versus three times per week. N. Engl. J. Med. 363, 2287-2300 (2010).

Competing interests statement

The authors declare no competing interests. 